\title{
Regional odontodysplasia: Account of clinical case in permanent teeth
}

\author{
Antonio Carrilho Neto ${ }^{*}$, Fábio Eduardo de Siqueira, Fernanda Michelli Martins, \\ Thaisa Bueno Godoy Parreira, Paula Mayumi Siqueira
}

State University of Londrina, Londrina, Brazil

Email: "ancarrilho@hotmail.com

Received 10 April 2013; revised 12 May 2013; accepted 9 June 2013

Copyright (c) 2013 Antonio Carrilho Neto et al. This is an open access article distributed under the Creative Commons Attribution License, which permits unrestricted use, distribution, and reproduction in any medium, provided the original work is properly cited.

\section{ABSTRACT}

The regional odontodysplasia, also referred as odontogenic dysplasia, is a rare condition which has been described as an alteration of the dental development affecting the mineralized structures of both the permanent and deciduous teeth, and it has been associated with retardation in the eruption of the affected teeth, gum edema, hydrocephalus and Epidermal Nevus Syndrome. The etiology is still unknown, however, some hypotheses have been suggested about their origin. About its incidence, this disease is more frequent in the anterior segment of the maxilla and usually unilateral. Epidemiologically this disease shows no predilection for any racial group, being more frequent in the first and second decade of life and predominantly in females. Clinically the Odontodysplasia Regional is characterized as an hypoplasia of enamel and dentin in teeth badly formed with size alteration and form coloration varying of the yellow to chestnut; the presence of smooth and extremely friable dentine, tooth mobility and defective mineralization increases the possibilities of the pulp exposure and a tendency to develop abscesses. Radiographically has the appearance of ghosts tooth with a bizarre morphology; reduction of the normal radiopacity decrease of the thickness of the dentine and enamel structures. They generally present with open apexes; shortened or absent roots; diffuse calcification in the inside of the pulp. Histopathologically it presents with accented reduction of the amount of dentin, presence of great interglobular dentine areas and irregular standard of the dentine canals. Enamel is usually thin, hypoplasic and hypocalcified. The pulp presents atrophic areas, fibrose and hyalinization and the cement does not have marked structural

"Corresponding author. alterations. The treatment of the Regional odontodysplasia has been the reason of many quarrels. This present essay aims to review the literature and report a clinical case.

Keywords: Teeth Ghosts; Odontodysplasia; Displasy Odontogenic; Imperfect Odontogenic

\section{INTRODUCTION}

It is a rare condition of dental development that affects the teeth mineralized structures, affecting as much the dentine as the enamel [1]. They can reach deciduous teeth and/or permanents [2-7]. It was initially described by MC Call \& Wald in 1947 [8]. In 1953 Shuter et al. [7] described the $1^{\text {st }}$ clinical case with clinical, hystologic and radiographic findings (pain, edema and fever). Rushton [9], in 1954, described the condition under the name "ghost tooth", and in 1963 Zegarelli et al. [10] were the first to use "odontodysplasia” term. Pindborg in 1970 added the prefix "regional” $[4,11]$.

In relation to the epidemiology of this pathology, Crawford \& Alfred [12] state that the age group of bigger prevalence is between the first and the second decade of life; without predilection for any racial group. In relation to the sex, the feminine one is more frequently attacked [3,6,13-16].

The biggest incidence is present in the anterior segment, being the maxilla two times more affected than jaw $[14,17]$. Generally, they are more frequent unilaterally $[15,17]$.

The etiology is still unknown, but some hypotheses have been raised as local ischemia $[1,18]$, teratogenic drugs [19], irradiation, vitaminic deficiencies, metabolic and nutricionais riots, local somatic mutation, premature degeneration of the agency of the enamel, latent virus inhabiting in the odontogenic epithelium that it becomes 
active during the development of teeth; incompatibility of $\mathrm{RH}$ factor $[1,7,9,10,12,18-25]$. There are no existed evidences of hereditary factors $[9,10,26]$, and trauma and previous infections had not been considered as etiologies $[6,16,27]$.

The regional odontodysplasia has been associated with retardation in the eruption of the affected teeth $[2,19,28]$ and gum swelling $[1,2,19,28,29]$, vascular nevus [3,30,31] and hydrocephfalus [32,33].

Clinicaly is characterized by hypoplasia of enamel and dentine in teeth badly formed with size and form alteration; coloration varying of the yellow to chestnut; presence of smooth and extremely friable dentine which is due to structural defects and defective mineralization, which increases the possibility of the pulp to be easily displayed with tendency to develop abcesses. They can present mobility for having roots badly formed and its eruption does not occur or can be partial, presenting modification in the chronology of eruption $[3,4,17,20$, 32,34-41].

Radiographycally, it has the appearance of ghosts with a bizarre morphology; reduction of the normal radiopacity because of the thickness of the dentine and enamel. They generally present open apexes; shortened or absent roots; diffuse calcification inside of the pulp; increase of the pulp chamber due to present thin walls with little dentine formation; the amelo-dentinal limit is absent [3-5, 9,10,16,19,31,35,40,42-45].

The histopatology characteristics of this condition are: accented reduction of the amount of dentine (poor mineralized); widening of the daily pay-dentine layer; presence of great interglobular dentine areas; irregular standard of the dentine canals; enamel is thin, hypoplasic and hypocalcified (it lacks amelogenesis). Characteristically the reduced epithelium of the enamel around of teeth that does not burst and present many irregular calcified bodies; absence of peritubular matrix. The pulp is presented with atrophic areas, fibrose and hyalinization with degenerative or vascularized odontoblasts; the cement does not have marked structural alterations $[2,4,6,7,9,14,19,24$, 46,47].

The treatment can be to extract the dysplasic teeth and to the confection of prosthesis $[4,19,27]$, or when the teeth erupt, can use prosthetic resource of dentistic (metallic restorations and/or crowns) to try to reestablish the patient aesthetically and functionally [13,36,44]. It should also be taken into account that the choice of treatment depends on the grade of dental development, aesthetic and functional needs, the patient's age, the number of teeth affected and the attitudes and desires of the child and parents [2-4,19].

\section{STORY OF CLINICAL CASE}

Patient M.H.G., 12 years, male, white, originating Lond-
rina/PR. He appeared to the Odontologyc Emergency Care Clinic of the State University of Londrina presenting mobility and painful symptomatology in element 21, with presence of abcess. Anamnesis did not disclose any story of systemic illness, nor local trauma. However, it was told that the child had a convulsion during childbirth and in the first 3 months after birth, the mother also said that the patient had the deciduous teeth affected.

To the clinical examination was evidenced a yellowish coloration in the elements 21, 22, 63, 24, 26 and 27; teeth with modified forms; partial eruption of elements 22, 63, 24, 26 and 27; Migration of the alveolar bone with anatomic alteration; accented occlusal consuming in elements 26 and 27; absence of mobility, with exception of the element to 21 (Figure 1). In the radiographic examination was detected a reduction of the radiopacity of enamel and dentine of the elements in question; bad formation and increase of the chamber pulp; radicular reabsorption of the elements 63 and 24; open apexes of elements 23, 25 and 26, and presence of abcess in element 26 (Figure 2); aspects of "ghost tooth" on the elements 23 and 25 (to see Figure 3).

The treatment was divided in three stages. The first one performed in the moment of the attendance in the Odontology Emergency Care Clinic by draining the abscess of the element 21 and by prescribing amoxicillin $500 \mathrm{mg}$ with intervals of 8 hours and posterior extraction of the element, due its extreme mobility. The second stage consisted in draining the abscess of the element 26

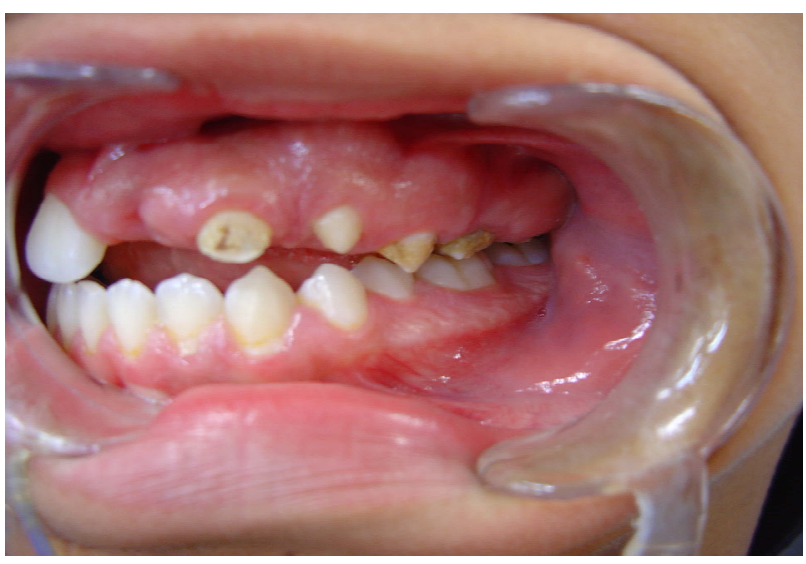

Figure 1. Clinical presentation of the intra-buccal examination.

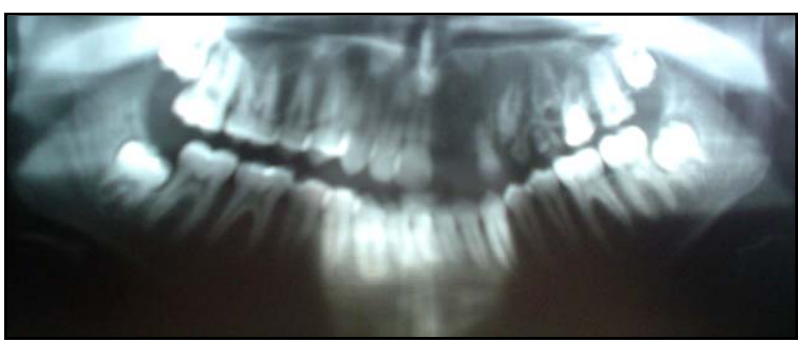

Figure 2. Examination of the panoramic radiography. 


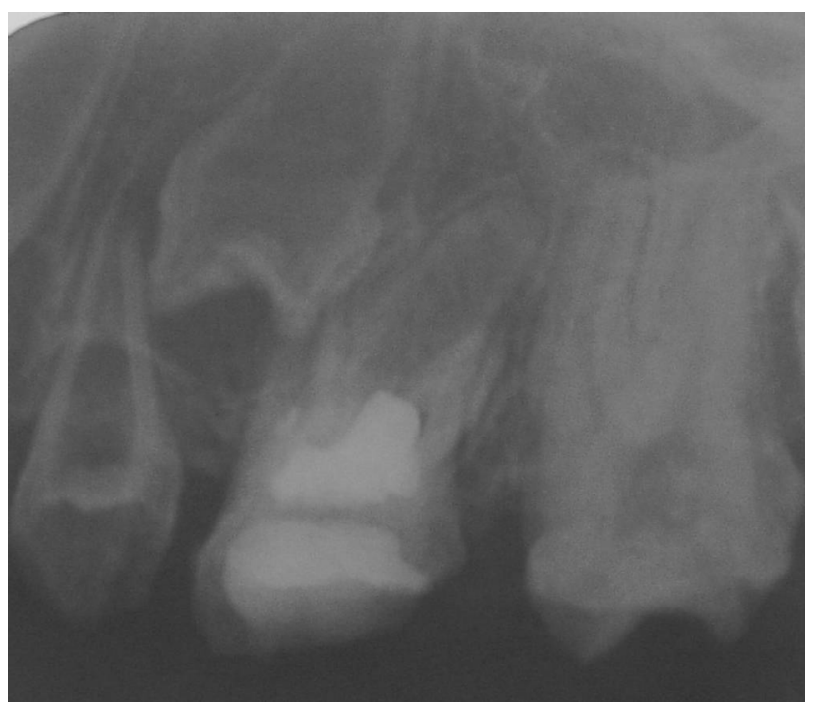

Figure 3. Examination of the periapical radiography of the elements 23, 25 and 26.

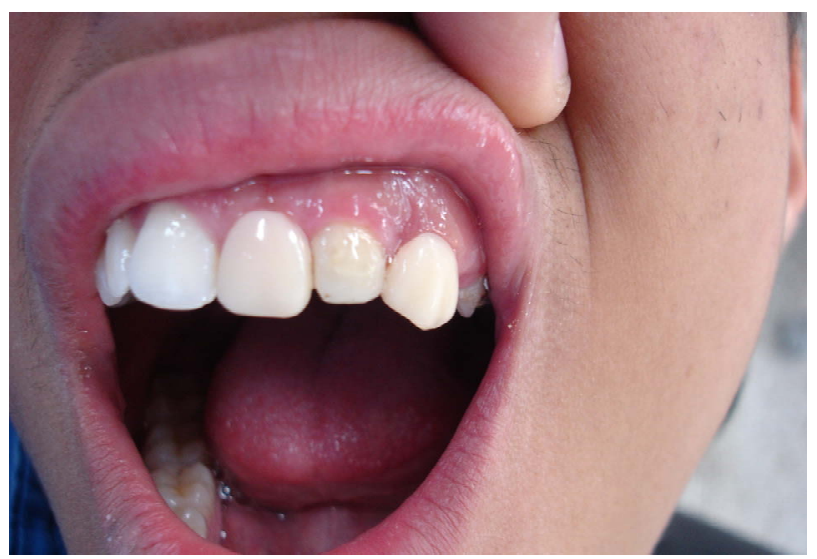

(a)

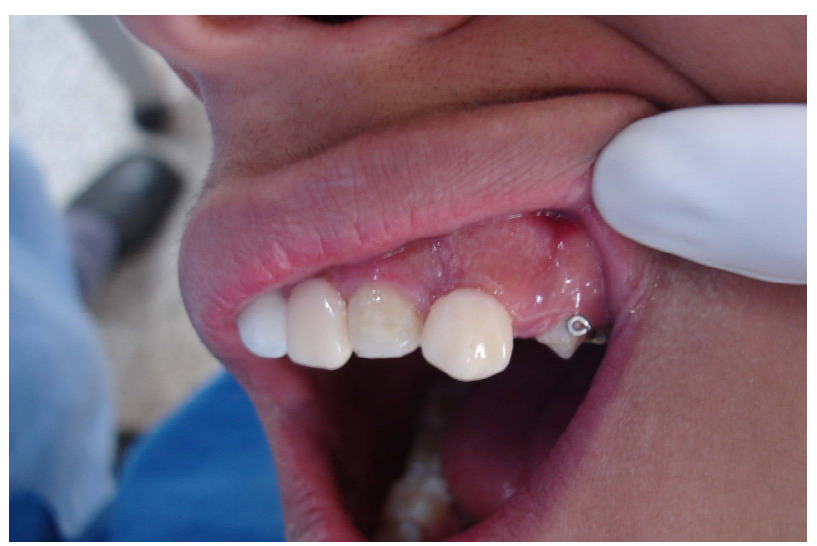

(b)

Figure 4. (a) Flexible Hawley Plate; (b) Flexible Hawley Plate, with palatal expander of $6.5 \mathrm{~mm}$ and adaptation of the absent elements (21, 23 and 24).

and posterior endodontic treatment. And the third stage was the confection of a flexible Hawley plate, with pala-

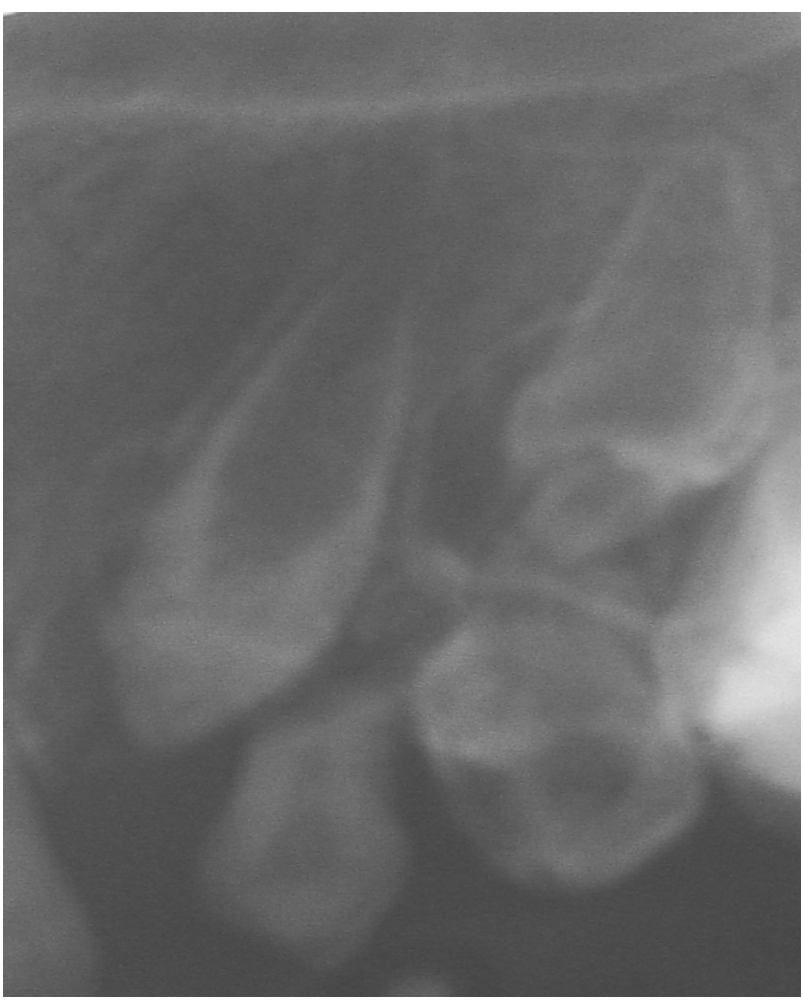

Figure 5. Radiographic exam demonstrating the aspects of the "ghost tooth".

tal expander of $6.5 \mathrm{~mm}$ and adaptation of the absent elements (21, 23 and 24) for one better aesthetic and functional reestablishment of the patient until he reaches the adult bone age for accomplishment of dental implants (Figures 4(a) and (b)).

\section{DISCUSSION}

The regional odontodysplasia is a rare pathological entity in which the enamel and the dentine are involved [9,14], its etiology is unknown. The prevalence occurs more frequently in the feminine sex, in the anterior region; and more frequently in the maxilla than in the jaw in a ratio of $2: 1$ [14].

Authors agree that the deciduous dentition and the permanent one can be affected, in the presented case both dentitions are involved. When the teeth affected exceeds the median line, the condition passes to be called as generalized odontodysplasia [13].

The treatment form has been the reason of many quarrels. Some authors say that the extraction of the involved elements and posterior confection of prosthesis would be the best option [27]. Another group of authors indicate dentistic procedures, if possible, for the aesthetic and functional reestablishment of the patient [13,36,44].

In the presented case, the treatment option was conservative, with the objective to preserve the structures affected until the patient reaches the adult age for poste- 
rior reevaluation and planning of possible implantations. The authors of this study did not agree with the conduct of who praise the extractions of all the affected elements, therefore, understanding of the maintenance of ghosts teeth in the intra-bone region will delay the displacement of the maxillary sinus in the occlusal direction, allowing the maintenance of the bone structure for future rank of implants (see Figure 5).

\section{REFERENCES}

[1] Burch, M.S., Besley, K.W. and Samuel, H.S. (1973) Regional odontodysplasia with associated midiline mandibular cyst: Report of case. Oral Surgery, Oral Medicine, Oral Pathology, 31, 44-48.

[2] Elisa, M.G., et al. (2009) Odontodysplasia in early childhood: A clinical and histological study. Journal of Indian Society of Pedodontics and Preventive Dentistry, 27, 175.

[3] Ganguly, R. and Ramesh, A. (2012) Journal of Indian Society of Pedodontics and Preventive Dentistry, 30, 176.

[4] Kirin, K. et al. (2010) Regional odontodysplasia. Journal of Indian Society of Pedodontics and Preventive Dentistry, 28, 315.

[5] Rezende, M.T.L., et al. (1998) Odontodisplasia regional em dentes permanentes. RGO, 46, 212-214.

[6] Shafer, W.G., Hine, M.K. and Lelvy, B.M. (1985) Distúrbios de desenvolvimento das estruturas bucais e parabucais. In: Shafer, W.G., Hine, M.K. and Lelvy, B.M., Eds., Tratado de Patologia Bucal, Interamerinaca, Rio de Janeiro, 57-58.

[7] Suher, T., Jump, E.B. and Landis, R.L. (1953) Localized arrested tooth development. Oral Surgery, Oral Medicine, Oral Pathology, 6, 1305-1314.

doi:10.1016/0030-4220(53)90262-X

[8] McCall, J.O. and Walds, S.S. (1952) Clinical dental roentgenology. 3rd Edition, Saunders, Philadelphia.

[9] Rushton, M.A. (1965) Odontodysplasia "ghost teeth". British Dental Journal, 119, 109-113.

[10] Zegarelli, E.V., et al. (1963) Odontodysplasia. Oral Surgery, Oral Medicine, Oral Pathology, 16, 187-193.

[11] Kappadi, D., et al. (2009) Regional odontodysplasia: An unusual case report. Journal of Oral and Maxillofacial Pathology, 13, 62.

[12] Crawford, P.J.M. and Alfred, M.J. (1989) Regional odontodysplasia: A bibliography. Oral Surgery, Oral Medicine, Oral Pathology, 18, 251-263.

[13] Ansare, G., et al. (1997) Regional odontdysplasia: Report of four cases. International Journal of Paediatric Dentistry, 7, 107-113.

[14] Lustman, H. and Ulmansky, M. (1976) Structural changes in odontodysplasia. Oral Surgery, Oral Medicine, Oral Pathology, 41, 193-202.

[15] Pandis, N., Polido, C. and Bell, W.H. (1991) Regional odontodysplasia. A case associed with asymmetric maxilary and mandibular development. Oral Surgery, Oral Medicine, Oral Pathology, 72, 492-496.

\section{doi:10.1016/0030-4220(91)90566-U}

[16] Pindborg, J.J. (1970) Pathology of the dental hard tissue. Saunders, Philadelphia, 435.

[17] Kahn, M.A., et al. (1991) Regional odontodysplasia. Oral Surgery, Oral Medicine, Oral Pathology, Oral Radiology and Endodontics, 72, 462-467.

[18] Lunin, M. and Devore, D. (1985) The etiology of regional odontodysplasia. J. Pedolont, 9, 233-243.

[19] Bailoor, D., et al. (2011) Journal of Indian Society of Pedodontics and Preventive Dentistry, 29, 323.

[20] Abrams, M. and Gropr, J. (1966) Odontodysplasia: Report of three cases. Journal of Dentistry for Children, 33, 353-362.

[21] Alexander, W.N., Lilly, G.E. and Irby, W.B. (1996) Odontodysplasia. Oral Surgery, Oral Medicine, Oral Pathology, 22, 814-820. doi:10.1016/0030-4220(66)90372-0

[22] Chaudhry, A.P., Wittich, H.C., Stickel, F.R. and Holland, M.R. (1961) Odontogenesis imperfecta: Report of a case. Oral Surgery, Oral Medicine, Oral Pathology, 14, 10991103. doi:10.1016/0030-4220(61)90503-5

[23] Fanibunda, K.B., et al. (1996) Odontodysplasia: Gingival manifestations and accompanying abnormalities. Oral Surgery, oral Medicine, Oral Pathology, Oral Radiology and Endodontics, 81, 84-88. doi:10.1016/S1079-2104(96)80154-5

[24] Marzola, C. and Damante, J.H. (1996) Odontodisplasia total. $R G O, \mathbf{3 4}, 140-143$.

[25] Sadeghi, E. and Ashrafi, M. (1981) Regional odontodysplasia: Clinical, pathologic and therapeutic considerations. JADA, 102, 336-339.

[26] Stafne, E.C. and Gibilisco, J.A. (1982) Anomalias. In: Stafne, E.C. and Gibilisco, J.A., Eds., Diagnóstico Radiográfico Bucal, 2nd Edition, Interamericna, Rio de Janeiro, pp. 31-32.

[27] O’Neil, D.W., Koch, M.G. and Lowe, J.W. (1990) Regional odontodysplasia: Report of case. Journal of Dentistry for Children, 74, 459-461.

[28] Hintz, C.S. and Peters, R.A. (1972) Odontodysplasia: Report of an unusual case and a review of the literature. Oral Surgery, Oral Medicine, Oral Pathology, 34, 744750. doi:10.1016/0030-4220(72)90292-7

[29] Gardner, D.J., et al. (1974) The dentinal changes in regional odontodysplasia. Oral Surgery, Oral Medicine, Oral Pathology, 38, 887-897. doi:10.1016/0030-4220(74)90341-7

[30] Sloatweg, P.J. and Meuwissen, P.R.M. (1985) Regional odontodysplasia in epidermal nevus syndrome. Journal of Oral Pathology \& Medicine, 14, 256-262.

[31] Walton, J.L., et al. (1978) Odontodysplasia. Oral Surgery, Oral Medicine, Oral Pathology, 92, 676-684.

[32] Cabral, et al. (1994) Regional odontodysplasia: A report of three cases. Quintessence International, 25, 141-145.

[33] Dahllof, G., et al. (1987) Concomitant regional odontdysplasia and hidrocephalus. Oral Surgery, Oral Medicine, Oral Pathology, 63, 354-357. doi:10.1016/0030-4220(87)90204-0 
[34] Adler, P. and Bencze, J. (1964) Amelogenesis imperfecta non hereditaria segmentalis. Dtsch. Zahnarztl. Z., 19, 1092-1104.

[35] Gardner, D.J. and Sapp, J.P. (1973) Regional odontodysplasia. Oral Surgery, Oral Medicine, Oral Pathology, 35, 351-356. doi:10.1016/0030-4220(73)90073-X

[36] Gerlach, R.F., et al. (1998) Regional odontodysplasia. Oral Surgery, Oral Medicine, Oral Pathology, Oral Radiology and Endodontology, 85, 308-313. doi:10.1016/S1079-2104(98)90014-2

[37] Hankey, G.T. and Duchwort, R. (1968) Odontodysplasia in the deciduous dentition. The Dental Practitioner and Dental Record, 19, 93-95.

[38] Lowry, L., et al. (1992) An unusual case of regional odontodysplaisa. International Journal of Paediatric Dentistry, 2, 171-176. doi:10.1111/j.1365-263X.1992.tb00031.x

[39] Neupert III, E.A. and Wright, J.M. (1989) Regional odontodysplasia presenting as a soft tissue swelling. Oral Surgery, Oral Medicine, Oral Pathology, 67, 193-196. doi:10.1016/0030-4220(89)90329-0

[40] Panella, J., et al. (1994) Odontodysplasia regional: Relato de um caso. Revista da Associação Brasileira de Odontologia-ABO Nacional, 2, 270-272.

[41] Pruhs, R.J., Simonsen, R.C., Sharma, P.S. and Fodor, B.J. (1975) Odontodysplasia. The Journal of the American Dental Association, 91, 1057-1066.

[42] Dayal, P.K., Dip, J. and Many, N.J. (1981) Odontodysplasia: Report of a case. Journal of Oral Medicine, 36, 79-81.

[43] Hovinga, J. and Ingenhoes, R. (1979) Regional odontodysplasia. International Journal of Oral Surgery, 8, 474477.

[44] Lowe, O. and Duperon, D. (1985) Generalized odontodysplasia. The Journal of Pedodontics, 9, 232-242.

[45] Miller, W.A. and Seymour, R.H. (1968) Odontodysplasia. British Dental Journal, 125, 56-59.

[46] Herald, R.C.B., Lally, E.T. and Gold, Z. (1976) Abnormal tooth tissue in human odondysplasia. Oral Surgery, Oral Medicine, Oral Pathology, 42, 357-365. doi:10.1016/0030-4220(76)90169-9

[47] Kerebel, B. and Kerebel, L.M. (1981) Enamel in odontodysplasia. Oral Surgery, Oral Medicine, Oral Pathology, 52, 404-410. doi:10.1016/0030-4220(81)90339-X 